\title{
Variabilidade interanual da profundidade óptica da atmosfera sobre Maceió, AL
}

\author{
Edivania de A. Lima ${ }^{1}$, Luis C. B. Molion ${ }^{2}$, Manoel F. Gomes Filho ${ }^{3}$, Janne L. da N. Firmino ${ }^{1}$ \& Alcides O. da Silva ${ }^{1}$
}

\begin{abstract}
RESUMO
A radiação solar de ondas curtas (ROC) que penetra na atmosfera, depende principalmente da turbidez atmosférica e da cobertura de nuvens. Realizou-se um estudo sobre a variabilidade da profundidade óptica da atmosfera sobre Maceió, AL, utilizando-se dados de radiação solar incidente no topo da atmosfera e emitida na superfície, de onda longa (ROL), cobertura de nuvens, água precipitável e precipitação, retirados do conjunto de dados de Reanálise do NCAR/NCEP. O ciclo anual da profundidade óptica estimada com esses dados foi comparado com os dados de radiação global medidos no Departamento de Meteorologia da UFAL (1997-1999). Nos meses de janeiro a junho de 1997, Maceió teve cerca de 60 a $80 \%$ de cobertura por nebulosidade e durante os meses de abril a julho, período chuvoso para Maceió, ocorreu maior cobertura de nuvens, cerca de 70 a $80 \%$ da cobertura do céu, aumentando a atenuação de ROC, sendo que as maiores concentrações de ROC foram verificadas no ano de 1999 (La Niña) com um máximo de $10 \mathrm{~mm} \mathrm{~d}^{-1}$ de precipitação. Os valores de transmissividade totalizadora calculados em função do espalhamento Ryleigh pelo vapor d'água, oscilaram de 0,30 a 0,47 .
\end{abstract}

Palavras chave: radiação solar, massa óptica, nebulosidade, turbidez

\section{Interannual variability of the optical depth of the atmosphere on Maceió, Alagoas}

\begin{abstract}
The short wave solar radiation (ROC) that passes through the atmosphere depends mainly on the atmospheric turbidity and cloud cover. A study of the variability of the optical depth of the atmosphere over Maceió, in the State of Alagoas, using incoming solar radiation in the top of the atmosphere and surface, was carried through long wave radiation (ROL), cloud covering, precipitable water and precipitation using the NCAR/NCEP re-analysis data set. The annual cycle of the optical depth obtained with these data, was compared through observed global radiation from the UFAL meteorology department's data set for the period from 1997 to 1999. In the period from January to June of the year 1997, Maceio had about 60 to 80 per cent of cloud cover and during the period from April to July (rainy season in that location) it had highest cloud cover of around 70 to 80 per cent, increasing the attenuation. The highest ROC concentration occurred in the year 1999 (La Niña conditions) with a maximum of $10 \mathrm{~mm}^{-1 a y}{ }^{-1}$ precipitation. The values of transmittance totalizer calculated in function of the Ryleigh scattering for the water vapor had oscillated from 0.30 to 0.47 .
\end{abstract}

Key words: solar radiation, optical depth, cloud covering, turbidity

1 Doutorando Meteorologia/UFCG, Av. Aprígio Veloso 882, Bodocongó, CEP 58109-970, Campina Grande, PB. Fone: (83) 3310-1054. E-mail: edy_al@hotmail.com; agsln@yahoo.com.br; alcidesolinto@hotmail.com

${ }^{2}$ Departamento de Meteorologia/UFAL, Cidade Universitária, Tabuleiro dos Martins, CEP 57072-970, Maceió, AL. Fone: (82) 3214-1365. E-mail: molion@radar.ufal.br

3 Unidade Acadêmica de Ciências Atmosféricas/UFCG. E-mail: mano@dca.ufcg.edu.br 


\section{INTRODUÇÃO}

A atmosfera pode ser considerada uma gigantesca máquina térmica, alimentada pela energia do Sol, que fornece 99,97\% da energia utilizada no sistema Terra Atmosfera, e provoca atenuação da radiação eletromagnética que a atravessa na faixa do espectro óptico. A radiação solar interage com a atmosfera terrestre por três processos físicos: processos de espalhamento por moléculas dos diferentes gases e aerossóis (Espalhamento Rayleigh); Espalhamento Mie; Absorção e reflexão, causadas pelos constituintes atmosféricos (gases, aerossóis e nuvens) (Reeves, 1975; Ometto, 1981; Garg, 1982; Novo, 1989).

Enquanto o espalhamento atmosférico e a reflexão simplesmente mudam a direção da radiação solar e dependem do comprimento de onda, a absorção, por outro lado é, em geral, seletiva, ou seja, alguns elementos não a absorvem em todo o seu espectro e, sim, em alguns comprimentos de onda preferenciais. O vapor d’água, o ozônio e o gás carbônico, são os principais agentes absorvedores, além desses gases, outros elementos também atuam na absorção da energia solar, como: $\mathrm{CH}_{4}, \mathrm{~N}_{2} \mathrm{O}, \mathrm{O}_{2}$, poeiras, bruma, partículas de carbono e gotículas de nuvens, dentre outros (Vianello \& Alves, 2000).

As partículas em suspensão na atmosfera também são capazes de absorver e dispersar radiação solar, o seu efeito é notável quando associado a fenômenos da intensidade de um incêndio florestal ou das cinzas expelidas por uma erupção vulcânica (Molion, 1994; Molion et al., 2001).

O Sol é um corpo essencialmente formado por plasma e sua atmosfera consiste de três camadas: fotosfera, cromosfera e coroa. A fotosfera é a camada que define o volume visível (superfície do Sol) (Echer et al., 2001); a energia solar é gerada por meio de complexas reações termonucleares, as quais convertem as partículas atômicas (prótons do núcleo de núcleo de hidrogênio) em partículas alfa (núcleo de hélio). Toda a energia emitida do Sol é na forma de radiação eletromagnética e para a radiação solar alcançar a superfície do solo, tem que atravessar a atmosfera terrestre, a qual é denominada massa óptica (Halacy Jr., 1977).

A Terra está envolvida pela atmosfera, que se constitui de moléculas de gases, poeiras em suspensão e outras partículas sólidas e líquidas, além de vários tipos de nuvens.

Para a radiação solar alcançar a superfície do solo, tem que atravessar a atmosfera terrestre, a qual é denominada massa óptica, onde a espessura ou profundidade óptica da atmosfera (EOA) é a medida da quantidade de matéria opticamente ativa atravessada por um feixe de radiação, que é avaliada, através da integração do coeficiente linear de atenuação ao longo do trajeto efetivamente percorrido pelo feixe de radiação ou, ainda, pela integração do coeficiente mássico de atenuação ao longo do respectivo caminho óptico (Hartmann, 1994).

A massa óptica que a radiação solar atravessa é unitária, isto é, a energia incidente na superfície (I) é igual à incidente no "topo" da atmosfera (Io), menos a parcela que sofreu extinção pela massa óptica (m).

De acordo com Stephens (1994), a profundidade óptica geralmente apresenta três componentes: uma devido ao espalhamento molecular de Rayleigh; outra relacionada com o espalhamento por aerossóis e, dependendo do comprimento de onda em questão, uma terceira componente, relacionada à absorção por certos gases traços, tais como ozônio.

As nuvens desempenham papel fundamental no balanço de energia do planeta visto que refletem e ainda absorvem intensamente a radiação solar e, em contrapartida, absorvem e reemitem a radiação de onda longa (infravermelha) térmica emitida pelos corpos terrestres, reduzindo o contraste entre o aquecimento diurno e o resfriamento noturno Sellers (1965).

Segundo Sellers (1965), cerca de 36\% do total de radiação solar interceptados pela Terra, retornam diretamente para o espaço por reflexão das nuvens, das partículas em suspensão no ar e da própria superfície.

A variação do albedo nuvens depende da variação da quantidade de vapor d’água e gelo contidos na nuvem e influencia mais a radiação difusa que a radiação direta; na maioria das vezes, a espessura da camada de nuvens é suficientemente grande para que tanto as nuvens claras como nuvens escuras reduzam quase a zero a radiação direta; quando o céu está limpo, a radiação direta corresponde de 60 a 87\% da radiação global (Hartmann et al., 1990).

De acordo com Echer et al. (2001), a radiação solar em condição de céu claro é pouco atenuada quando comparada com condições de céu encoberto (parcialmente ou totalmente). Nuvens e aerossóis atuam como elemento espalhador, principalmente da radiação solar. Como a região de comprimentos de onda mais curtos apresenta maior componente de radiação difusa em relação à radiação de ondas longas, a presença de nuvens reduz muito mais a região do visível que a região do ultravioleta.

O conhecimento da intensidade da radiação solar incidente em uma superfície quer seja horizontal ou ortogonal aos raios solares, têm aplicabilidade em diversas áreas como engenharia, arquitetura, meteorologia, agricultura e hidrologia. Em termos agrícolas, a radiação solar é responsável por processos fotobioquímicos dos vegetais, os quais estão ligados às quantidades de horas às exposições ao brilho solar, além de determinar a época de floração, frutificação e interferir na qualidade dos frutos e grãos.

Analisou-se, neste a possível relação entre a cobertura de nuvens, quantidade de água precipitável na atmosfera e os processos atenuantes da radiação solar incidente na superfície da Terra, sob a forma de radiação direta e difusa.

\section{MATERIAL E MÉTODOS}

Os dados mensais meteorológicos (cobertura de nuvens (tcdc (\%))), radiação de onda longa-ROL (ulwrf $\left(\mathrm{MJ} \mathrm{m}^{-2}\right)$ ), água precipitável (pw_wtr $\left(\mathrm{kg} \mathrm{m}^{-2}\right)$ ), e precipitação (prate $\left(\mathrm{mm} \mathrm{dia}{ }^{-1}\right)$ ) usados neste trabalho, foram extraídos do National Centers for Environmental Prediction National Center for Atmospheric Research (NCEP-NCAR) do projeto Reanalysis (Kalnay et al., 1996). Esses dados foram tratados e visualizados através do GrADS (Grid Analysis and Display System, versão: 2.8 SL, referenciado por (Doty, 1992)), por 
meio de cartas de distribuição horizontal. Em adição aos dados acima, usaram-se séries temporais de radiação solar direta e radiação difusa, medida em dois piranômetros da KIPP e ZONEM, modelo CM5, sendo que a componente difusa foi obtida colocando-se um anel de sombreamento sobre um dos solarímetros, ambos instalados no Departamento de Meteorologia da Universidade Federal de Alagoas, ( $9^{\circ} 40^{\prime} \mathrm{S}$; $35^{\circ} 42^{\prime} \mathrm{W}$ e $127 \mathrm{~m}$ de altitude) e dados de constante solar $\left(\mathrm{Wm}^{-2}\right)$ medidos pelo satélite ERBS (The Earth Radiation Budget Satellite). Os dados de constante solar $\left(\mathrm{Wm}^{-2}\right)$, medidos pelo ERBS, foram obtidos através de valores individuais e totais da irradiância solar que representam média das medidas instantâneas, corrigidas para o ângulo entre a linha central óptica do instrumento e o sol e normalizadas com relação à distância Terra-Sol. A cada duas semanas foram coletados medidas com intervalo de 64 segundos de uma coleta para outra, correspondendo ao primeiro intervalo de dados; posteriormente, esse intervalo foi subdividido em dois intervalos de 32 segundos. As medidas das resultantes dos dois períodos distintos foram utilizadas para definir a irradiância solar fora da atmosfera terrestre).

Geraram-se, de início, médias climatológicas (janeiro de 1997 a dezembro de 1999) para as variáveis meteorológicas selecionadas; essas médias foram calculadas como as médias aritméticas dos valores de precipitação, radiação de onda longa (ROL), e nebulosidade para cada ponto de grade e para cada dia do ano, segundo a fórmula:

$$
\bar{P}_{i}=\frac{1}{N} \sum_{j=1}^{N} P_{i, j} \quad i=1,366 ; \quad j=1, N
$$

donde $\mathrm{P}_{\mathrm{i}, \mathrm{j}}$ é uma grade latitude - longitude para o dia "i” $\mathrm{e}$ para o ano “j”, $\overline{\mathrm{P}}_{\mathrm{i}}$ são os valores médios de cada uma das quatro variáveis consideradas em cada ponto de grade para o dia "i” e $\mathrm{N}$ é o número de anos utilizados, em seguida, foram calculadas as anomalias para cada ponto de grade, sendo estas definidas simplesmente pela diferença entre a variável total e a média calculada acima, esse procedimento foi feito para permitir a construção de diagramas de Hovmoller a fim de se estudar uma eventual propagação de sinais, enquanto as variáveis foram usadas durante o período para realçar as comparações entre os referentes dados do NCEP e os demais fornecidos pelo Departamento de Meteorologia da Universidade Federal de Alagoas.

Construiu-se, com os dados do satélite ERBS, uma tabela com os máximos valores mensais da constante solar que, inicialmente, estavam em $\mathrm{Wm}^{-2}$ e foram integrados ao período diurno resultando em total diário de energia em $\left(\mathrm{MJ} \mathrm{m}^{-2}\right)$ por meio da multiplicação do valor original pela quantidade de horas brilho solar do período e por $3600 \mathrm{~s}$, posteriormente, selecionaram-se os maiores valores de radiação global ( $\mathrm{MJ} \mathrm{m}^{-2}$ ), dentre os dados medidos em superfície para a elaboração do cálculo da transmissividade totalizadora $(\tau)$ média diária. A transmissividade totalizadora $(\tau)$ foi calculada a partir da razão entre o fluxo de ROC obtido na superfície e integrado no período diurno e o fluxo, também integrado no período diurno, obtido pelo ERBS no topo no topo da atmosfera.
Para o cálculo da transmitância espectral para absorção molecular pelos gases uniformemente misturados na atmosfera $\left(\mathrm{CO}_{2}, \mathrm{~N}_{2}, \mathrm{O}_{2}\right.$, etc.), utilizou-se o algoritmo, baseado no código para computador, denominado LOWTRAN, desenvolvido por pesquisadores do Laboratório de Pesquisa da Força Aérea dos Estados Unidos em Cambridge (Iqbal, 1983).

$$
\tau_{\mathrm{g} \lambda}=\exp \left[-1,41 \mathrm{k}_{\mathrm{g} \lambda} /\left(1+118,93 \mathrm{k}_{\mathrm{g} \lambda} \mathrm{m}_{\mathrm{a}}\right)^{0,45}\right]
$$

donde $\mathrm{k}_{\mathrm{g} \lambda}$ é o coeficiente de atenuação para a mistura de gases e $m_{a}$, sua massa óptica. A transmitância espectral correspondente do vapor d’água é:

$$
\tau_{\mathrm{w} \lambda}=\exp \left[-0,2385 \mathrm{k}_{\mathrm{w} \lambda} \mathrm{wm}_{\mathrm{w}} /\left(1+20,07 \mathrm{k}_{\mathrm{w} \lambda} \mathrm{wm}_{\mathrm{w}}\right)^{0,45}\right]
$$

donde $k_{w \lambda}$ é o coeficiente de atenuação, $m_{w}$ a massa óptica do vapor d’água, e w a água precipitável dada em centímetros. Os valores dos coeficientes de atenuação constam de tabelas existentes, por exemplo, em Iqbal (1983).

Para cálculo da atenuação da radiação solar global, integrada em todos os comprimentos de onda, para céu claro em Maceió, considerando-se a proximidade do equador, podese adotar a massa óptica igual à unidade para a mistura de gases e para o vapor d'água, isto é, $\mathrm{m}_{\mathrm{g}}=\mathrm{m}_{\mathrm{w}}=1,00$. O coeficiente $\mathrm{k}_{\mathrm{g} \lambda}$ foi feito igual a zero, resultando em uma transmissividade $\tau_{\mathrm{g} \lambda}=1,00$; para a mistura de gases e para a absorvidade do vapor d'água $\left(\alpha_{\mathrm{w}}\right)$ adotou-se a formulação proposta por Lacis \& Hansen, apud Iqbal (1983), que é função da água precipitável.

$$
\alpha_{\mathrm{w}}=2,9 \mathrm{w} /\left[(1+141,5 \mathrm{w})^{0,635}+5,925 \mathrm{w}\right]
$$

Para atenuação devido ao espalhamento Rayleigh usou-se uma expressão desenvolvida por Davies (1980), apud Iqbal, (1983), tal que a transmitância $\left(\tau_{\mathrm{R}}\right)$ se reduz a:

$\mathrm{R}=0,972-0,08262 \mathrm{ma}+0,00933 \mathrm{ma}^{2}-0,00095 \mathrm{ma}^{3}+0,000437 \mathrm{ma}^{4}$

\section{RESULTADOS E DISCUSSÃO}

Observa-se, na Figura 1 distribuição temporal da nebulosidade sobre a faixa longitudinal compreendida entre 50 a $30^{\circ} \mathrm{W}$, em que a cidade de Maceió ( $\left.35^{\circ} \mathrm{W}\right)$, está localizada. Nota-se que durante o ano de 1997 a cobertura de nuvens variou de 20 a $80 \%$ o que representa uma atenuação maior da radiação de onda curta devido ao maior caminho óptico percorrido pela energia solar.

A partir do final de julho de 1997 ao mês de outubro do mesmo ano, período no qual a atmosfera se encontra mais limpa, como se previa a cobertura de nuvens decresceu cobrindo cerca de 20 a 50\% do céu; logo, nesta situação o principal fator atenuante da irradiância solar é a variação do ângulo de elevação do Sol e não a cobertura de nuvens (Baldocchi et al., 1984). Comparando-se os meses de julho a outubro de 1997 com o mesmo período de 1998, observou-se que, neste ano, a cobertura de nuvens apresentou percentagem menor (10 a 40\%) em relação à cobertura de nuvens, observada no ano de 1997 (20 a 50\%), ou seja, durante o 
ano de 1998 (nesses meses) a atmosfera se apresentou menos úmida que no mesmo período de 1997.

Em 1999, observou-se que o comportamento da cobertura de nuvens sobre Maceió foi similar ao observado durante o ano de 1998. Fazendo-se uma comparação entre todo o período analisado, notou-se que o ano de 1997 foi o que apresentou a menor percentagem de nebulosidade, $20 \%$, sobre a cidade de Maceió, fato este que pode ser atribuído ao fenômeno global El Nino que provoca, sobre a região do Nordeste do Brasil, forte subsidência, ou seja, menor formação de nuvens, pois de acordo com o Instituto Nacional de Pesquisas Espaciais (INPE), o fenômeno (El Niño 1997/98) evoluiu rapidamente desde abril de 1997 (Infoclima, 1998).

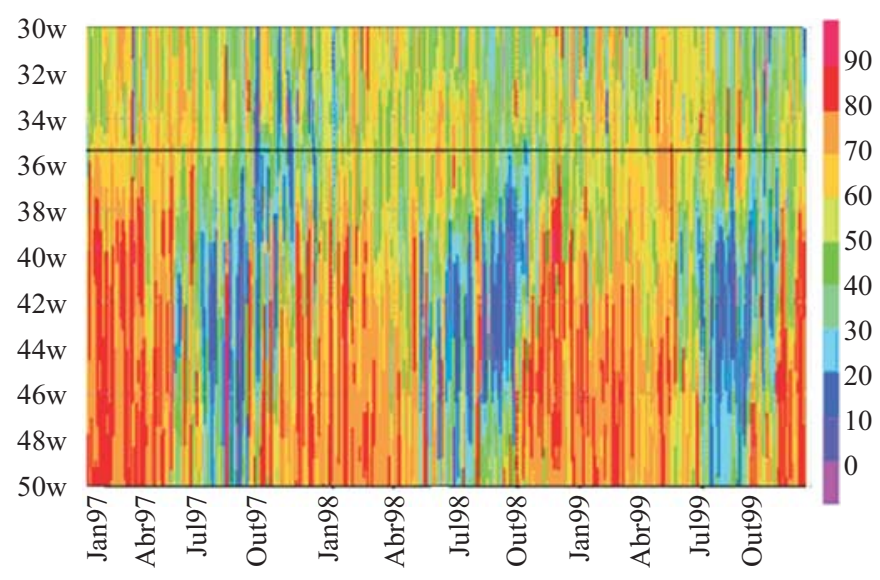

Figura 1. Evolução temporal da cobertura de nuvens (\%) e média diária, para a latitude de $10^{\circ} \mathrm{S}$, período de janeiro 1997 a dezembro 1999

A evolução temporal do fluxo de radiação de onda longa emitida para o espaço (Figura 2), analisada na faixa compreendida entre 30 e 50 w, durante os anos de 1997 a 1999. Observam-se, nas proximidades da cidade de Maceió (assinalada por uma reta), os núcleos de mínimos de ROL (19 $\mathrm{MJ} \mathrm{m}^{-2}$ a $22 \mathrm{MJ} \mathrm{m}^{-2}$ ) ocorreram nos meses de janeiro a abril ao longo do período analisado, e que o ano de 1997 apresentou os menores fluxos de radiação de onda longa com um mínimo de $18 \mathrm{MJ} \mathrm{m}^{-2}$. Os fluxos máximos de radiação de onda longa emitida para o espaço sobre Maceió ocorreram nos meses de julho a outubro em todo o período analisado; observou-se, também, que o ano de 1997 foi marcado com o máximo de ROL (26 $\mathrm{MJ} \mathrm{m}^{-2}$ ) durante todo o período analisado.

Comparando-se a Figura 2 com a Figura 1, notou-se que durante o período em que foram registrados os menores fluxos de radiação de onda longa a cobertura de nuvens sobre a cidade de Maceió obteve uma percentagem variando de 60 a $80 \%$ de nuvens, ou seja, maior cobertura de nuvens implica em uma diminuição de emissão de ROL, uma vez que os topos das nuvens altas refletem mais radiação direta diminuindo a incidência da mesma na superfície e, conseqüentemente, a emissão de ROL (Lestrade, 1990).

Nota-se, na Figura 3, a evolução temporal da precipitação em mm/dia, para a cidade de Maceió, durante o período compreendido de janeiro de 1997 a dezembro de 1999.

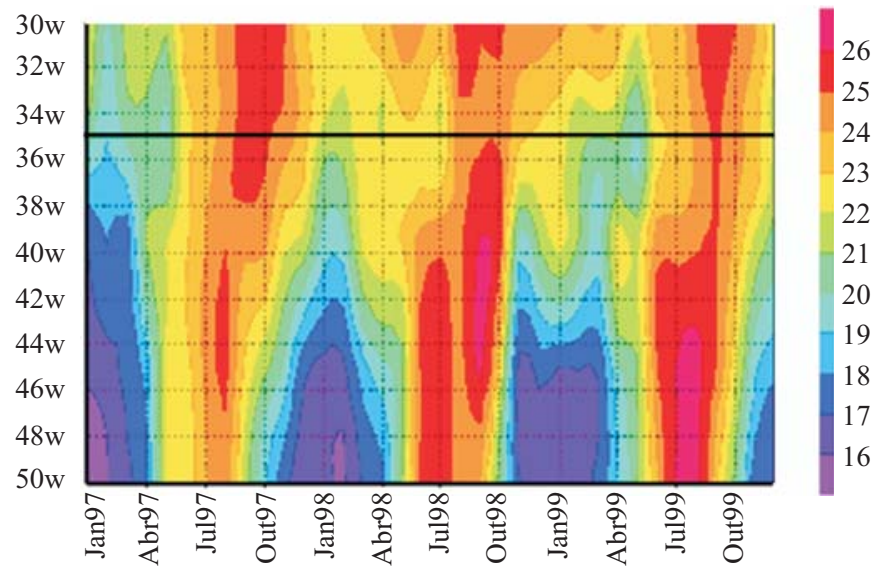

Figura 2. Variação temporal do fluxo de radiação de onda longa $\mathrm{MJ} \mathrm{m}^{-2}$ para a latitude de $10^{\circ} \mathrm{S}$, no período de janeiro de 1997 a dezembro de 1999

Os valores de precipitação durante os meses de janeiro a abril de 1997, variaram de 6 a $10 \mathrm{~mm} \mathrm{dia}^{-1}$, constatandose o mesmo intervalo de meses para o ano de 1998, além de um decréscimo em relação ao mínimo valor atingindo $3 \mathrm{~mm} \mathrm{dia}^{-1}$.

Em termos gerais, durante o ano de 1997 (El Nino) foram observados os menores totais pluviométricos sobre a cidade de Maceió, isto é, durante os anos de 1997 a 1999; segundo Figueiredo (2002) ocorre aqui à estação chuvosa da cidade (abril a julho), no ano de 1999, justamente que apresentou os maiores totais de chuvas verificadas durante todo o período de estudo.

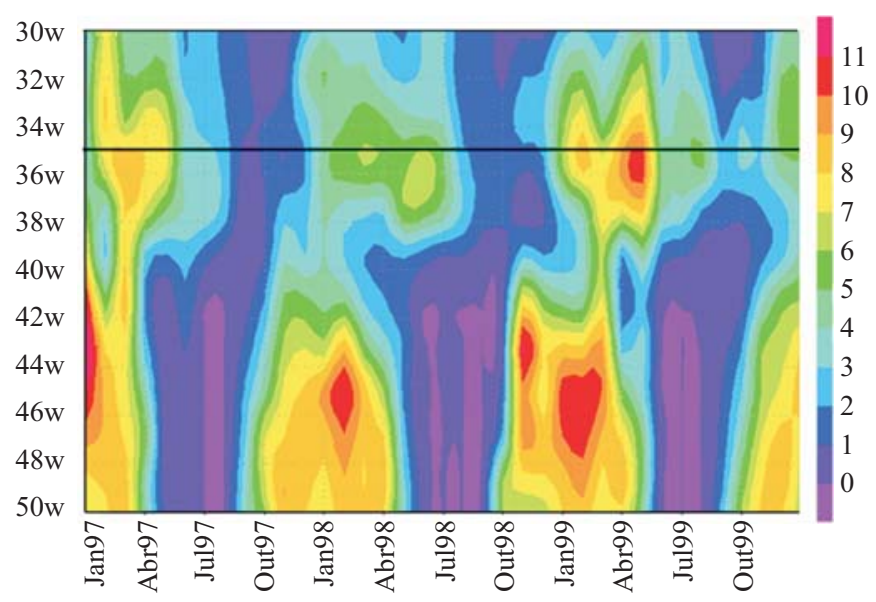

Figura 3. Evolução temporal da precipitação média mensal ( $\left.\mathrm{mm} \mathrm{dia}^{-1}\right)$ para a latitude de $10^{\circ} \mathrm{S}$, período de janeiro 1997 a dezembro 1999

De acordo com a fórmula proposta por Iqbal (1983) foram calculados os valores de absorvidade que a energia solar sofre pelo efeito do vapor d'água. Observou-se que o maior valor de absorvidade ocorreu nos meses de março de 1997 e maio de 1999 com o valor de 0,144 enquanto o menor valor $(0,129)$, se deu nos meses de agosto e setembro de 1997. Notou-se, para a cidade de Maceió, que no período dos maiores índices pluviométricos (abril a julho) os valores de absorvidade oscilaram de 0,137 a 0,144 , porém 
para o período em que a atmosfera se encontrou mais limpa, os valores estiveram compreendidos entre 0,129 a 0,138 (setembro a dezembro).

Os valores de transmissividade total $(\tau)$, obtidos através do produto entre a transmissividade do vapor d’água $(\tau \mathrm{w})$ e a transmissividade do espalhamento Rayleigh $(\tau R)$ ficaram compreendidos entre 0,771 (janeiro de 1997), e 0,779 (dezembro de 1999), nos meses de abril a julho, os valores de transmissividade variaram de 0,773 (abril, 1998) a 0,779 (julho, 1999) neste intervalo de tempo se esperava que os valores de $\tau \mathrm{R}$ fossem menores devido à maior espessura óptica que ocorre durante os meses, com maior quantidade de precipitação.

Vê-se, na Figura 4, valores de transmissividade total $(\tau)$, calculados em função do espalhamento Ryleigh, pelo vapor d'água. O ciclo anual da transmissividade totalizadora evidencia que o menor valor ao longo do período estudado, ocorreu no mês de março no ano de 1997 e maio de 1999, com valor de 0,770 . Na evolução do ciclo anual percebe-se que o pico de máxima transmissividade se deu no ano de 1997 para os meses de agosto e setembro de 1997, com o valor de 0,784 , nota-se, ainda, uma constância nos valores de transmissividade para o mês de novembro, nos anos de 1997 a 1999, com o valor de 0,778. Durante o ano de 1997 os valores de transmissividade variaram de 0,771 (janeiro) a 0,784 (agosto); nos meses de janeiro a abril, os valores oscilaram de 0,771 a 0,774 e tornaram crescentes até o mês de agosto $(0,784)$ e setembro. Similarmente, no ano de 1998 os valores decresceram de setembro a dezembro atingindo o mínimo de 0,776 em dezembro.

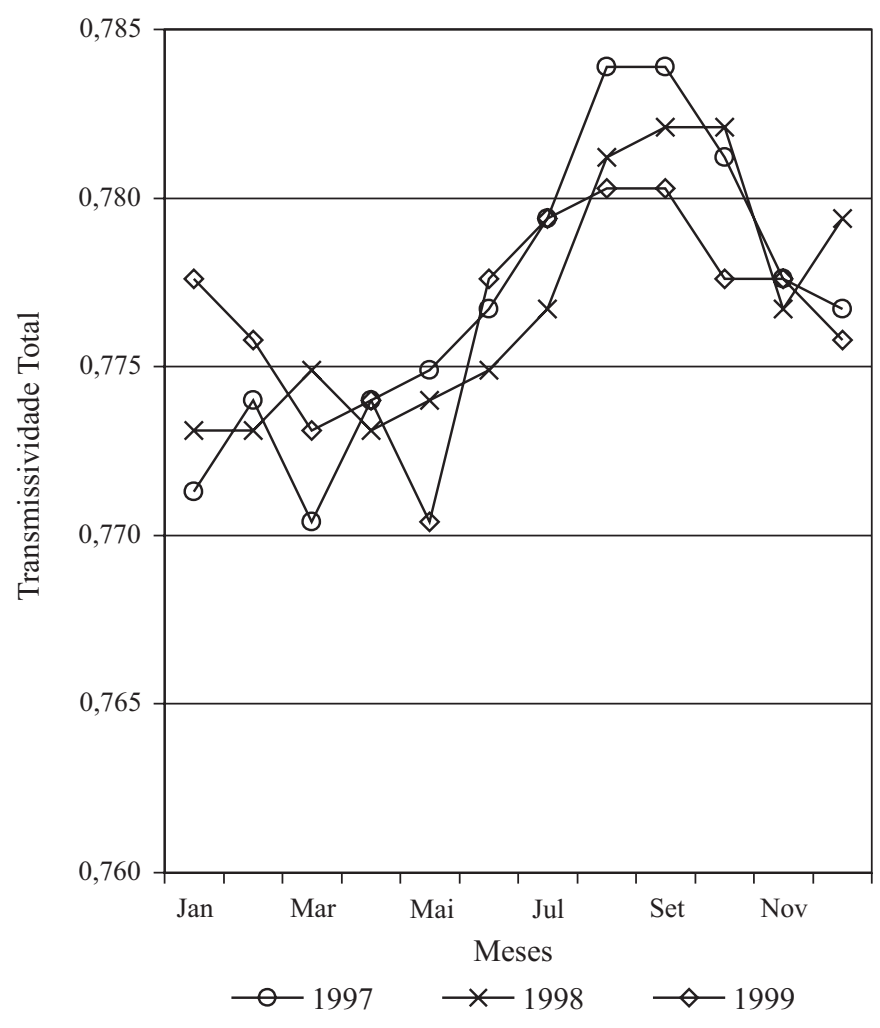

Figura 4. Gráfico da distribuição anual da transmissividade totalizadora calculada $(\tau)$

\section{CONCLUSÕES}

1. A intensidde de radiação solar, quer seja na forma direta ou difusa, atinge a superfície da Terra dependerá da variabilidade natural da transmissão atmosférica e dos processos atenuantes que nela ocorrem.

2. A percentagem de nebulosiade existente em determinada região é fator de grande importância nos processos atenuantes da radiação solar, visto que o clima, em escalas global, regional e local, é fortemente influenciado pela cobertura de nuvens e suas propriedades ópticas que, por sua vez, dependem de suas propriedades microfísicas.

3. A avaliação dos totais pluviométricos quando se deseja observar a variabilidade da profundidade óptica de uma localidade, torna-se primordial pois a quantidade de vapor d’água existente na atmosfera influencia nos processos de transmissividade atmosférica.

4. É de extrema necessidade o conhecimento sobre a quantidade de radiação solar que atinge a superfície da terra, visto que um dos principais enfoques da radiação solar é, atualmente, o seu aproveitamento para a produção alternativa de energia elétrica. As fontes convencionais de geração energética mais utilizada no mundo, como petróleo e gás natural, são não-renováveis em curto prazo, de tal forma a energia proveniente do Sol é a viabilizadora de todos os processos de geração energética, quer seja hidráulica, por dar origem ao ciclo hidrológico; eólica, por gerar um gradiente de pressão provocado pelo aquecimento diferencial da superfície terrestre, termoelétrica, bem como em tempos remotos os combustíveis fosséis e biomassa, por serem os vegetais captadores e transformadores dessa energia, através da fotossíntese.

\section{LITERATURA CITADA}

Baldocchi, D.; Jacovides, C. P.; McCree, K. J. Seasonal variations in the radiation regime within an oak-hickory forest. Agricultural For Meteorology, Amsterdam, v.33, n.5, p.177191, 1984.

Doty, P. Federal Research and Development (R\&D) as intellectual property, United States Scientific and Technical Information: Views and perspectives. Norwood A blex, v.5, n.6, p.139-171, 1992.

Echer, E.; Sousa M. P.; Schuch, N. J. The beer's law applied to the earth's atmosphere. Revista Brasileira de Física, São Paulo, v.23, n.3, p.276-283, 2001.

Figueiredo, J. O. G. R. Distribuição espacial da pluviometria em Alagoas com ênfase a variabilidade causada pelos padrões de temperatura da superfície do mar. Maceió: UFAL, 2002. 148p. Dissertação Mestrado

Garg, H. P. Tratise on solar energy. Fundamentals of solar energy. 1.ed. Chichester: Jonh Wiley and Sons, 1982.587p.

Halacy Jr., D. S. Earth, water, wind and sun: Our energy alternatives. New York: Haper e Row, 1977.186p.

Hartmann, D. L. Global physical climatology international geophysics series, Pratice Hall, New York, v.56, n.4, p.4079, 1994. 
Hartmann, H. T.; Kester, D. E.; Davies Jr., F. T. Plant propagation: principles and practices. 5.ed. New York: Prentice Hall, 1990. 647p.

Infoclima. Boletim de informações climáticas. Ano 5.1998. http:// www.cptec.inpe.br/ products/climanalise/infoclima/ 15 Jul. 2001.

Iqbal, M. An introduction to solar radiation. 2.ed. Londres: Academic, 1983. 390p.

Kalnay, E.; Kanamitsu, M.; Kistler, R. The NCEP/NCAR 40 year reanalysis project. Bulletim of American Meteorological Society, New York, v.77, p.437-471. 1996.

Lestrade, J. P. The effect of cloud layer plane albedo on global and diffuse isolation solar energy. Elmsford, New York, v.44, n.22, p.115-121, 1990.

Molion, L. C. B, Efeito dos vulcões no clima. Caderno de geociências, Rio de Janeiro, v.12, n.10, p.13-23, 1994.

Molion, L. C. B.; Querino, C. A. S.; Menezes, D. C.; Lima, E. A.; Silva, H. O.; Borba, J. C. C. Possíveis efeitos de aerossóis vul- cânicos no balanço radiativo da reserva biológica nacional do Jarú na Amazônia. In: Congresso Brasileiro de Meteorologia, 12, 2001, Foz do Iguaçu. Anais... Foz do Iguaçu: SBMET, 2001. CD-Rom

Novo, E. M. L. M. Sensoriamento remoto: princípios e aplicações 1.ed. São Paulo: Edgard Blucher, 1989. 308p.

Ometto, J. C. Bioclimatologia vegetal. 1.ed. São Paulo: Agronômica Ceres, 1981. 425p.

Reeves, R. G. Manual of remote sensing. Falls Church: American Society of Photogrammetry, v.1, 1975. 867p.

Sellers, W. D. Physical Climatology, 1.ed. New York: Sellers, W.D. Physical Climatology. University of Chicago, 1965. 203p. Stephens, G. L. Remote sensing of the lower atmosphere. Oxford: Oxford University Press, 1994. cap.6, p.261-269.

Vianello, R. L.; Alves, A. R. Meteorologia básica e aplicações. 1.ed. Viçosa: Imprensa Universitária, 1991. 304p. 